\title{
Caracterização microestrutural de compósitos de hidroxiapatita-alumina confeccionados pelo método da esponja polimérica
}

\section{(Microstructural characterization of hydroxyapatite-aluminum oxide composites made by the polymeric sponge method)}

\author{
M. S. Abreu, A. G. S. Galdino \\ Instituto Federal de Ensino, Pesquisa e Extensão do Espírito Santo, Av. Vitória, 1729, Jucutuquara, \\ Vitória, ES 29040-780 \\ moniqsales@gmail.com,andregsg@ifes.edu.br
}

\begin{abstract}
Resumo
O aumento do número de cirurgias de substituição e reparação óssea propiciou o estudo e a disseminação do uso de biomateriais, devido à sua biocompatibilidade e custo inferior ao de outros materiais utilizados como implantes. A hidroxiapatita é um biomaterial eficaz para utilização em engenharia tecidual, por ela fazer parte da matriz óssea. Porém, sua baixa resistência mecânica torna necessária a adição de outros materiais objetivando aumentar tal propriedade. A alumina é um material bioinerte que tem sido bastante utilizado como reforço mecânico para a hidroxiapatita. Este trabalho buscou desenvolver, pelo método da esponja polimérica, arcabouços cerâmicos de hidroxiapatita-alumina nas proporções em massa $50 \%-50 \%, 60 \%-40 \%$ e $70 \%$-30. Os corpos de prova foram calcinados a $550{ }^{\circ} \mathrm{C}$ e sinterizados a 1250,1300 e $1350{ }^{\circ} \mathrm{C}$ para avaliar características microestruturais. Os resultados obtidos estão de acordo com a literatura, o que justifica a realização de ensaios de caracterização físico-mecânica.

Palavras-chave: biocerâmicas, hidroxiapatita, alumina, método da esponja polimérica.
\end{abstract}

\begin{abstract}
The increase in number of surgeries for bone exchanging and repairing allowed researches and use of biomaterials due to their biocompatibility and low cost when compared to other materials used as implants. Hydroxyapatite is one of the most used material for tissue engineering because it is part of the bone matrix. However, hydroxyapatite has low mechanical strength and it is necessary to mix it with other materials to improve that property. Alumina is a bioinert material which has been used as mechanical reinforcement for hydroxyapatite. This research aimed to develop hydroxyapatite-alumina ceramic scaffolds by the polymeric sponge method, with compositions of 50\%-50\%, 60\%-40\% and 70\%-30\% by weight. The samples were calcined at 550 ${ }^{\circ} \mathrm{C}$ and sintered at 1250, 1300 and $1350{ }^{\circ} \mathrm{C}$ for microstructural analysis. The results were in accordance to literature, and it is recommended to do physical-mechanical essays.
\end{abstract}

Keywords: bioceramics, hydroxyapatite, alumina, polymeric sponge method.

\section{INTRODUÇÃO}

O aumento dos problemas de estrutura óssea, provenientes de processos inflamatórios, acidentes ou doenças inerentes ao envelhecimento humano, despertou nos últimos anos a preocupação dos pesquisadores em investigar materiais substitutos de elevada pureza química e que não produzam reações adversas nos tecidos vivos, tornando necessário o desenvolvimento de suplentes funcionais para o tecido danificado a partir da engenharia tecidual, que aplica os princípios da biologia e da engenharia [1-4]. Os biomateriais utilizados na engenharia óssea devem apresentar propriedades físicas e biológicas compatíveis com o organismo vivo hospedeiro, provocando a reação adequada do mesmo. Dentre os biomateriais utilizados, sobretudo as biocerâmicas à base de fosfato de cálcio, é crescente a aplicação da hidroxiapatita para modificação e restauração de defeitos ósseos [2-4].
Além da biocompatibilidade, o biomaterial utilizado na engenharia tecidual óssea deve apresentar propriedade osteoindutiva ou osteocondutiva e permitir o crescimento ósseo, sendo gradualmente substituído por um novo tecido. Neste contexto, a hidroxiapativa se destaca por ser um material biocompatível, não carcinogênico, não alergênico, seguro e clinicamente admissível na reconstrução de defeitos ósseos [3-5]. Porém, a fragilidade e a baixa resistência às tensões mecânicas da hidroxiapatita têm limitado seu uso em intervenções ortopédicas [6, 7]. A fim de incorporar propriedades mecânicas à hidroxiapatita (HA), sem afetar sua biocompatibilidade, tem-se estudado a formação de compósitos de hidroxiapatita agregada à alumina $\left(\mathrm{Al}_{2} \mathrm{O}_{3}\right)$, que se trata de um material bioinerte e com alta resistência às tensões mecânicas [8]. Por se tratar de um material bioativo, a hidroxiapatita é gradualmente substituída pelo osso [9], enquanto a alumina, por ser bioinerte, não causa nenhum tipo de imunorreação no paciente e não 
afeta a biocompatibilidade da hidroxiapatita [8]. Este trabalho buscou desenvolver composições de compósitos de hidroxiapatita-alumina nas proporções em massa 50\% $\mathrm{HA}-50 \% \quad \mathrm{Al}_{2} \mathrm{O}_{3}, 60 \% \quad \mathrm{HA}-40 \% \quad \mathrm{Al}_{2} \mathrm{O}_{3}$ e $70 \%$ HA-30\% $\mathrm{Al}_{2} \mathrm{O}_{3}$, e caracterizar suas microestruturas. A escolha dessas proporções foi baseada nos resultados físico-mecânicos obtidos no trabalho desenvolvido por [10].

\section{MATERIAIS E MÉTODOS}

Materiais: utilizou-se hidroxiapatita (HA) cedida pela empresa Inside Materiais Avançados, Belo Horizonte, MG. Objetivando identificar os percentuais de elementos químicos presentes na mesma, foram realizados ensaios de espectroscopia de fluorescência de raios X (FRX) em um espectrômetro da Rigaku RIX 3100. Os resultados do ensaio de FRX estão indicados na Tabela I. A alumina (APC-G) foi cedida pela empresa Alcoa, Poços de Caldas, MG. A análise química foi realizada pelo fornecedor, e encontra-se indicada na Tabela II. A esponja polimérica utilizada foi uma esponja comercial de poliuretano com densidade 28. A escolha da esponja baseou-se no tamanho que os poros deveriam ficar após sofrer a retração linear no processo de sinterização. A micrografia da esponja utilizada está ilustrada na Fig. 1. Para tal micrografia, foi utilizado o microscópio eletrônico de varredura Jeol JXA 840A. Observou-se que o formato dos poros foi arredondado e com tamanho superior a $100 \mu \mathrm{m}$, que é o tamanho mínimo para que seja possível hospedar componentes celulares e extracelulares dos ossos, além dos vasos sanguíneos [3]. Foram utilizados bicarbonato de sódio da Sigma-Aldrich Lot\# 011M01471, álcool etílico P.A. - ACS da Dinâmica Química Contemporânea e água destilada. $\mathrm{O}$ bicarbonato de sódio foi utilizado na formação do compósito por ser um agente ligante e defloculante.

Tabela I - Resultados de espectroscopia de fluorescência de raios $\mathrm{X}$ da hidroxiapatita, como recebida.

[Table I - Results of X-ray fluorescence spectroscopy of as received hydroxyapatite.]

\begin{tabular}{cc}
\hline Elemento & $\begin{array}{c}\text { Quantidade } \\
(\% \text { em massa })\end{array}$ \\
\hline $\mathrm{O}$ & 45,428 \\
$\mathrm{Ca}$ & 37,168 \\
$\mathrm{P}$ & 17,018 \\
$\mathrm{Mg}$ & 0,131 \\
$\mathrm{Sr}$ & 0,083 \\
$\mathrm{Si}$ & 0,051 \\
$\mathrm{Al}$ & 0,045 \\
$\mathrm{Fe}$ & 0,022 \\
$\mathrm{~S}$ & 0,019 \\
$\mathrm{Na}$ & 0,017 \\
$\mathrm{~K}$ & 0,013 \\
$\mathrm{Ni}$ & 0,005 \\
\hline
\end{tabular}

Tabela II - Resultados da análise química da alumina APC-G, como recebida.

[Table II - Results of chemical analysis of as received APC-G alumina.]

\begin{tabular}{cc}
\hline Componente & $\begin{array}{c}\text { Quantidade } \\
\text { (\% em massa })\end{array}$ \\
\hline $\mathrm{Al}_{2} \mathrm{O}_{3}$ & 99,600 \\
$\mathrm{SiO}_{2}$ & 0,029 \\
$\mathrm{Fe}_{2} \mathrm{O}_{3}$ & 0,029 \\
$\mathrm{Na}_{2} \mathrm{O}$ total & 0,120 \\
\hline
\end{tabular}

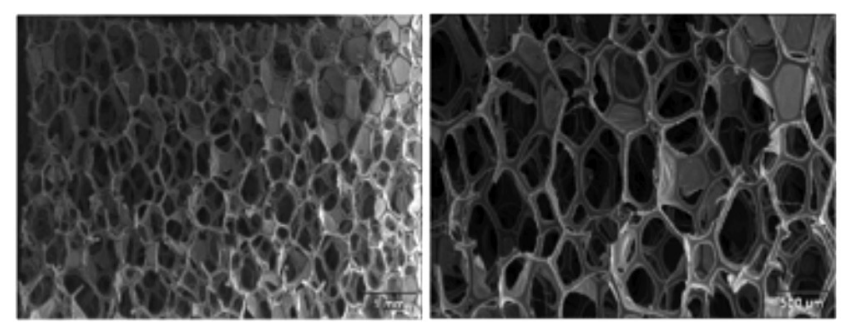

Figura 1: Micrografias obtidas por microscopia eletrônica de varredura da esponja comercial de poliuretano: (a) visão geral da esponja; (b) ilustra geometria e interconectividade dos poros.

[Figure 1: SEM micrographs of commercial polyurethane sponge: (a) general view of the sponge; (b) shows pores geometry.]

Métodos: para a confecção dos corpos de prova, utilizouse o método da esponja polimérica [11]. Inicialmente, a hidroxiapatita e a alumina foram misturadas em três proporções mássicas diferentes, a saber: 50\% $\mathrm{HA}-50 \% \mathrm{Al}_{2} \mathrm{O}_{3}$ (A1) $60 \% \mathrm{HA}-40 \% \mathrm{Al}_{2} \mathrm{O}_{3}$ (A2) e $70 \% \mathrm{HA}-30 \% \mathrm{Al}_{2} \mathrm{O}_{3}$ (A3). Após essa mistura, adicionou-se o bicarbonato de sódio, uma vez que esse atua como agente ligante e floculante. Para a formação da barbotina, utilizou-se uma solução composta por $40 \%$ de álcool e $60 \%$ de água em volume. Essa solução foi colocada em um béquer e em seguida adicionou-se a massa cerâmica composta por $\mathrm{HA}-\mathrm{Al}_{2} \mathrm{O}_{3}$ e bicarbonato de sódio. Após agitação, a barbotina foi formada. Foram preparados corpos de prova de poliuretano com dimensões de $10 \mathrm{~mm}$ de diâmetro por $10 \mathrm{~mm}$ de altura, que foram submergidos na barbotina e secaram na temperatura ambiente. Foram confeccionados cinco corpos de prova por composição para avaliação em três diferentes temperaturas. Estes foram aquecidos a $550{ }^{\circ} \mathrm{C}$ a $0,5{ }^{\circ} \mathrm{C} / \mathrm{min}$ para a retirada da esponja polimérica, mantidos por $1 \mathrm{~h}$ nessa temperatura e, em seguida, sinterizados a 1250,1300 e $1350{ }^{\circ} \mathrm{C}$ a $10^{\circ} \mathrm{C} / \mathrm{min}$, mantidos nessas temperaturas por $2 \mathrm{~h}$. Para a volatilização da esponja polimérica foi utilizado um forno EDG FA-IV e para a sinterização dos corpos de prova utilizou-se um forno Lindberg Blue M Thermo Scientific. O resfriamento até a temperatura ambiente aconteceu dentro do forno. As amostras foram submetidas a ensaios de caracterização microestrutural via microscopia eletrônica de varredura (MEV), Shimadzu SSX-550, e difração de raios X (DRX), difratômetro Bruker, D2 Phaser. A análise de DRX foi realizada com tensão de 40 kV / 20 mA com radiação incidente de CuK $\alpha$ e feixe difratado monocromatizado por um monocromador 
curvo de grafita. As amostras foram medidas no intervalo de $2 \theta$ de 20 a $80^{\circ}$ no modo de varredura contínua com velocidade de $1 \% / \mathrm{min}$ com passo de $0,02^{\circ}$ à temperatura ambiente $\left(23^{\circ} \mathrm{C}\right)$. Por MEV observou-se a morfologia dos poros e grau de sinterização das amostras. Por DRX observou-se as fases cristalinas presentes nos compósitos.

\section{RESULTADOS E DISCUSSÃO}

Após a sinterização a 1250,1300 e $1350{ }^{\circ} \mathrm{C}$, os corpos de prova da amostra $\mathrm{A} 1$ não mantiveram a integridade física, e com isso não foram considerados nos resultados apresentados. Isso se deveu ao fato da alumina não sinterizar bem nas temperaturas utilizadas para esse experimento. Com isso, não houve densificação adequada para os corpos porosos, levando à falência dos arcabouços. A temperatura de fusão da alumina é $2050{ }^{\circ} \mathrm{C}$ [10] e a da hidroxiapatita é $1760{ }^{\circ} \mathrm{C}$ [10]. A temperatura de sinterização especificada é normalmente da ordem de $66 \%$ a $75 \%$ da temperatura de fusão do composto [12], o que coloca a temperatura de sinterização da alumina dentro de um intervalo de temperatura entre 1367 a $1537{ }^{\circ} \mathrm{C}$, onde os melhores resultados se apresentam em temperaturas mais elevadas, uma vez que há mais difusão atômica, preenchendo a região de contato interpartículas, levando à densificação do material [13]. Com isso, quanto maior for a quantidade percentual de alumina nesse compósito e também por ele ser poroso, os corpos porosos do compósito $50 \% \mathrm{HA}-50 \% \mathrm{Al}_{2} \mathrm{O}_{3}$ não mantiveram a integridade física.

Os corpos de prova das amostras A2 e A3 mantiveram a integridade física após a sinterização a 1250,1300 e $1350{ }^{\circ} \mathrm{C}$, permitindo a realização das análises microestruturais através de MEV e de DRX. Os resultados de MEV realizada nos corpos de prova confeccionados pelo método da esponja polimérica para as amostras A2 e A3 sinterizadas a 1250, 1300 e $1350{ }^{\circ} \mathrm{C}$ estão ilustrados nas Figs. 2 a 5. As Figs. 2 e 3 ilustram as estruturas porosas obtidas pelo método da esponja polimérica para as amostras A2 e A3 sinterizadas nas temperaturas de trabalho do artigo. Como se pode observar, o corpo cerâmico reproduziu a estrutura física da esponja polimérica, que foi volatilizada a $550{ }^{\circ} \mathrm{C}$. As Figs. 4 e 5 ilustram o aspecto das partículas após sinterização, onde se pode observar que, a $1250^{\circ} \mathrm{C}$, o corpo cerâmico não foi bem sinterizado, pois houve presença de partículas pequenas e não houve formação de placas e agregados; a 1300 e
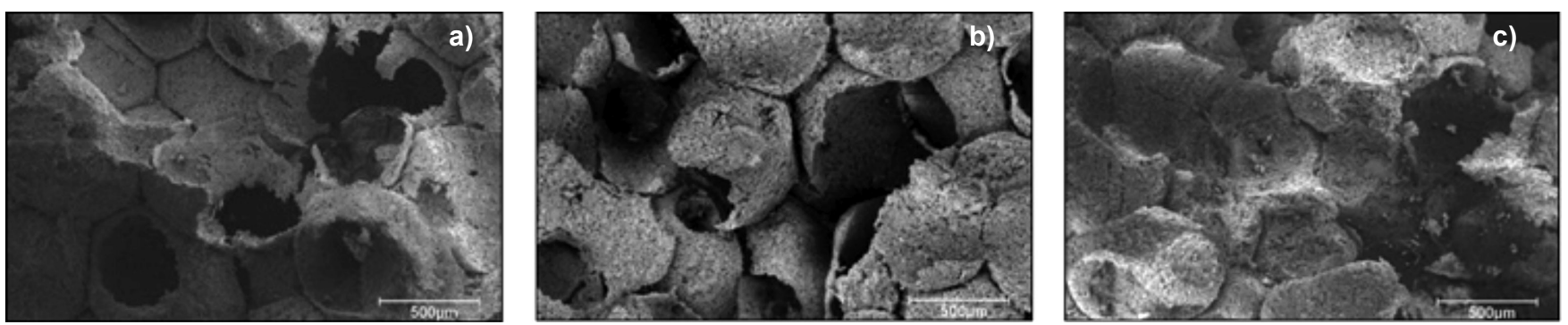

Figura 2: Micrografias obtidas por microscopia eletrônica de varredura da amostra $\mathrm{A} 2$ sinterizada a: (a) $1250{ }^{\circ} \mathrm{C}$, (b) $1300{ }^{\circ} \mathrm{C}$ e (c) $1350{ }^{\circ} \mathrm{C}$. [Figure 2: SEM micrographs of sample A2 sintered at: (a) $1250^{\circ} \mathrm{C}$, (b) $1300{ }^{\circ} \mathrm{C}$, and (c) $1350{ }^{\circ} \mathrm{C}$.]
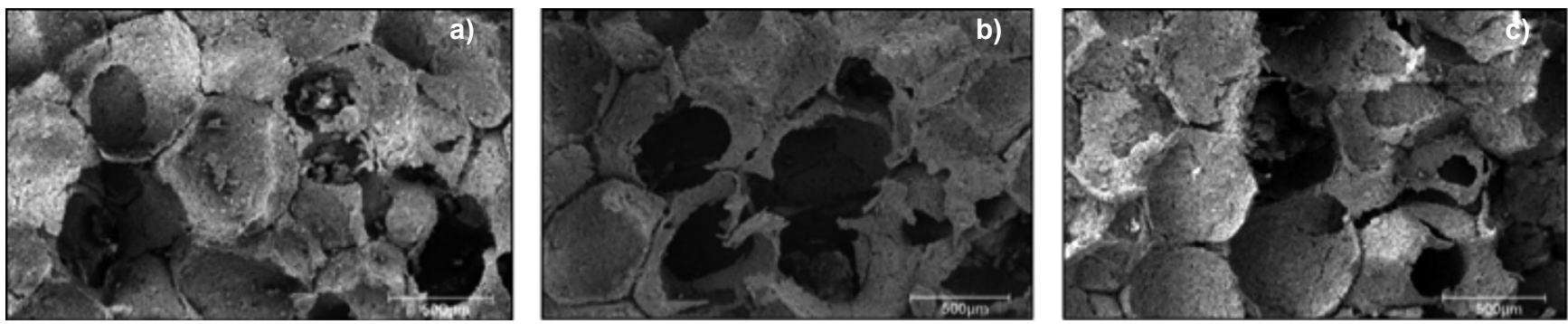

Figura 3: Micrografias obtidas por microscopia eletrônica de varredura da amostra $\mathrm{A} 3$ sinterizada a: (a) $1250{ }^{\circ} \mathrm{C}$, (b) $1300{ }^{\circ} \mathrm{C}$ e (c) $1350{ }^{\circ} \mathrm{C}$. [Figure 3: SEM micrographs of sample A3 sintered at: (a) $1250^{\circ} \mathrm{C}$, (b) $1300{ }^{\circ} \mathrm{C}$, and (c) $1350^{\circ} \mathrm{C}$.]
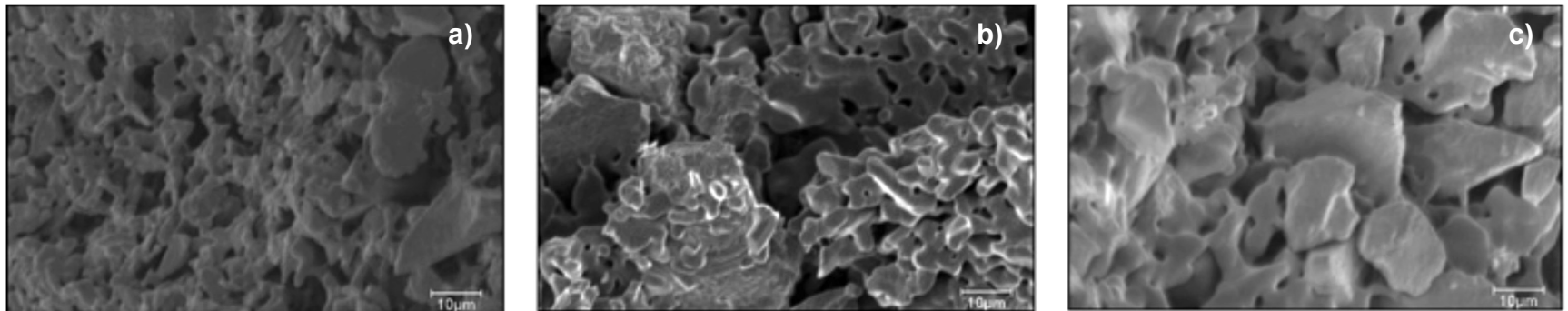

Figura 4: Micrografias obtidas por microscopia eletrônica de varredura mostrando detalhes da amostra $\mathrm{A} 2$ sinterizada a: (a) $1250{ }^{\circ} \mathrm{C}$, (b) $1300{ }^{\circ} \mathrm{C}$ e (c) $1350{ }^{\circ} \mathrm{C}$.

[Figure 4: SEM micrographs showing details of sample A2 sintered at: (a) $1250^{\circ} \mathrm{C}$, (b) $1300{ }^{\circ} \mathrm{C}$, and (c) $1350{ }^{\circ} \mathrm{C}$.] 

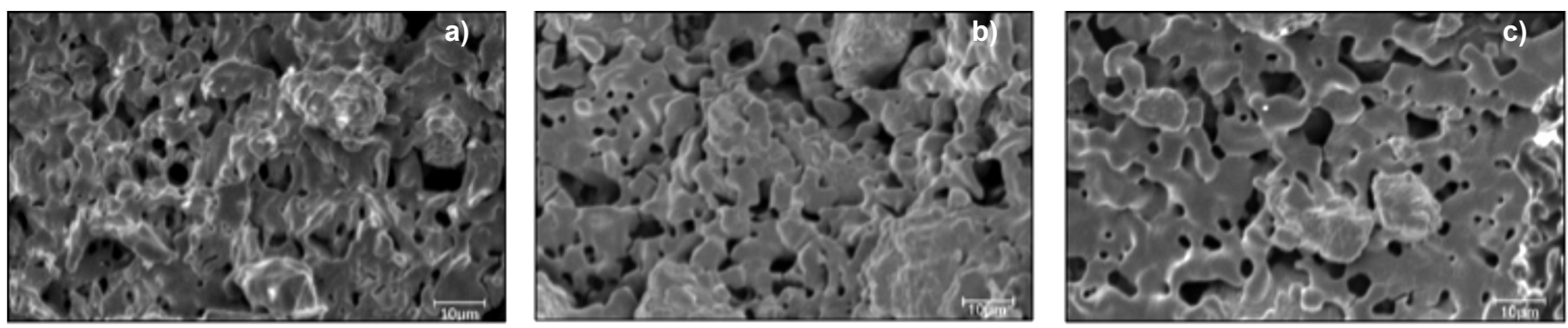

Figura 5: Micrografias obtidas por microscopia eletrônica de varredura mostrando detalhes da amostra $\mathrm{A} 3$ sinterizada a: (a) $1250{ }^{\circ} \mathrm{C}$, (b) $1300{ }^{\circ} \mathrm{C}$ e (c) $1350{ }^{\circ} \mathrm{C}$.

[Figure 5: SEM micrographs showing details of sample A3 sintered at: (a) $1250^{\circ} \mathrm{C}$, (b) $1300{ }^{\circ} \mathrm{C}$, and (c) $1350^{\circ} \mathrm{C}$.]

$1350{ }^{\circ} \mathrm{C}$, houve indícios de uma melhor sinterização, com aumento no tamanho do grão e maior formação de placas e agregados, com menos presença de partículas pequenas.

Os difratogramas apresentados nas Figs. 6 e 7 foram obtidos a partir das amostras A2 e A3 preparadas pelo método da esponja polimérica após sinterização a 1250 , 1300 e $1350{ }^{\circ} \mathrm{C}$. O ensaio foi realizado com as amostras na forma de pó. Os difratogramas permitiram observar que houve transformação de fases, onde a hidroxiapatita, após a sinterização dos corpos de prova, apresentou-se na forma alotrópica fosfato de cálcio $\left[\mathrm{Ca}_{3}\left(\mathrm{PO}_{4}\right)_{2}\right]$, além da reação entre a alumina e a hidroxiapatita, na forma de $\mathrm{CaAl}_{4} \mathrm{O}_{7}$ (grossita) e $\mathrm{CaAl}_{2} \mathrm{O}_{4}$. Também se observou que não houve resíduos da esponja polimérica nas duas composições após sinterização em três temperaturas diferentes. Para a análise de difração de raios X, foram utilizados os arquivos 01-088-0826 para a
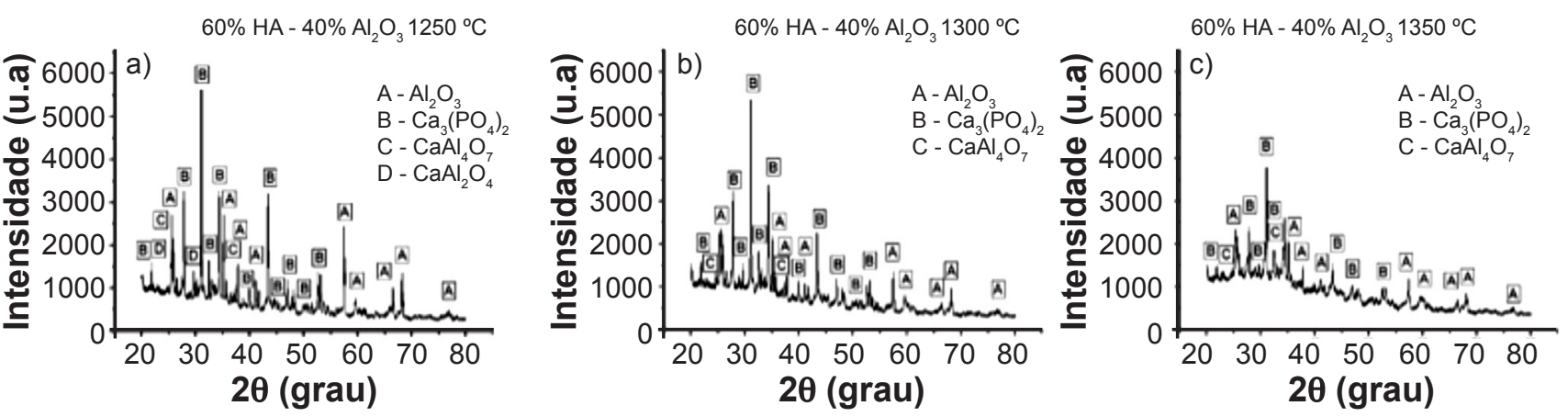

Figura 6: Difratogramas de raios $\mathrm{X}$ da amostra A2 sinterizada a: (a) $1250{ }^{\circ} \mathrm{C}$; (b) $1300{ }^{\circ} \mathrm{C}$; (c) $1350{ }^{\circ} \mathrm{C}$.

[Figure 6: XRD patterns of sample A2 sintered at: (a) $1250^{\circ} \mathrm{C}$; (b) $1300^{\circ} \mathrm{C}$; (c) $\left.1350{ }^{\circ} \mathrm{C}.\right]$
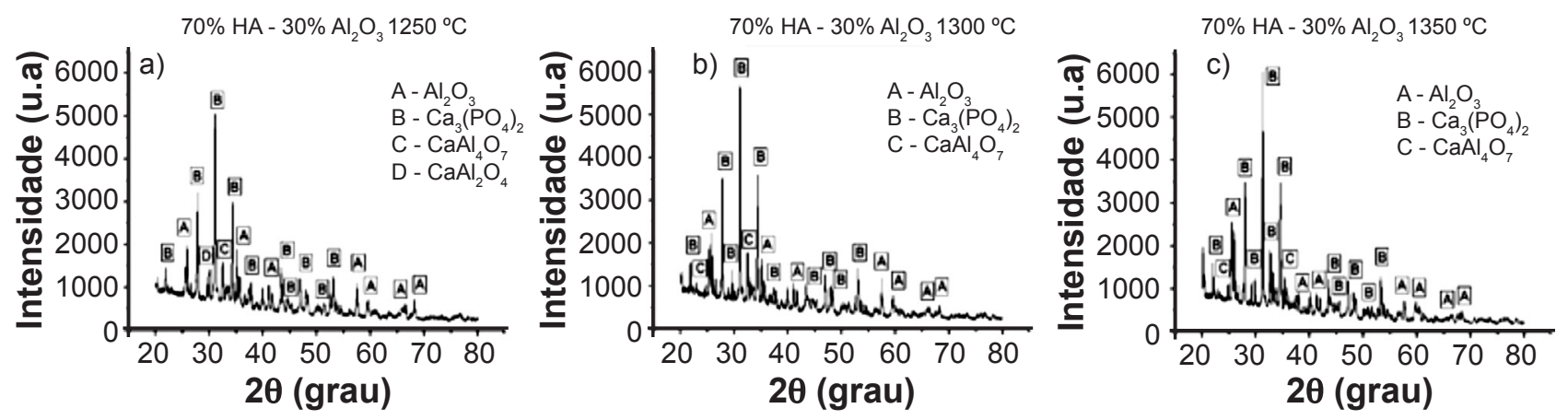

Figura 7: Difratogramas de raios X da amostra A3 sinterizada a: (a) $1250{ }^{\circ} \mathrm{C}$; (b) $1300^{\circ} \mathrm{C}$; (c) $1350{ }^{\circ} \mathrm{C}$.

[Figure 7: XRD patterns of sample A3 sintered at: (a) $1250^{\circ} \mathrm{C}$; (b) $1300^{\circ} \mathrm{C}$; (c) $1350{ }^{\circ} \mathrm{C}$.]

alumina, 01-070-2065 para o fosfato de cálcio, 00-046-1475 para a grossita e 00-034-0440 para o $\mathrm{CaAl}_{2} \mathrm{O}_{4}$. Também foi possível perceber a presença dos picos principais da alumina e do fosfato de cálcio, com leves alterações nas intensidades dos mesmos, o que indicou modificação nos parâmetros de rede cristalina. Esses valores de intensidade dos picos estão de acordo com a literatura [10].

\section{CONCLUSÕES}

Os resultados obtidos nas análises microestruturais permitiram concluir que a melhor condição para aplicações de crescimento ósseo foi a da amostra com $70 \%$ hidroxiapatita e $30 \% \mathrm{Al}_{2} \mathrm{O}_{3}$ sinterizada a $1350{ }^{\circ} \mathrm{C}$, por ter apresentado melhor sinterização, além da manutenção do formato 
estrutural da esponja polimérica após sinterização, embora as outras condições também possam ser utilizadas (limite de resistência à compressão, microdureza, toxicidade, etc.), baseadas no critério de tamanho de poros. Porém, ainda é necessária a realização de caracterização físico-mecânica dessas composições, a fim de estabelecer melhoria nas propriedades do compósito quando comparadas com as da hidroxiapatita, uma vez que a hidroxiapatita possui resposta fraca sob tensão mecânica.

\section{AGRADECIMENTOS}

Os autores agradecem ao INCT-Biofabris pela doação dos reagentes químicos, à Alcoa S/A pela alumina APC-G, à Inside Ltda. pela hidroxiapatita, ao Labiomec/DEMA/FEM/ UNICAMP pela utilização dos equipamentos, ao CEFETMG pela utilização do microscópio eletrônico de varredura para análise microestrutural.

\section{REFERÊNCIAS}

[1] E.Y. Kawachi, C.A. Bertran, R.R. Reis, O.L. Alves, Quím. Nova 23, 4 (2000) 518-522.

[2] A.C.F.M. Costa, M.G. Lima, L.H.M.A. Lima, V.V. Cordeiro, K.M.S. Viana, C.R. Souza, H.L. Lira, REMAP 4, 3 (2009) 29-38.

[3] R.L. Oréfice, M.M. Pereira, H.S. Mansur, "Biomateriais fundamentos e aplicações", Cultura Médica, Rio de Janeiro,
RJ (2006) 59-81.

[4] B.D. Ratner, A.S. Hoffman, F.J. Schoen, J.E. Lemons, Biomaterials science an introduction to materials in medicine, Academic Press, San Diego, CA, EUA (1996) 73 83.

[5] H.Y. Juang, M.H. Hon, Mater. Sci. Eng. C 2 (1994) 7781.

[6] D.M. Liu, Key Eng. Mater. 115 (1996) 209-232.

[7] L.R. Rodrigues, M. Motisuke, C.A.C. Zavaglia, Anais $21^{\circ}$ Congresso Brasileiro de Engenharia Biomédica, Salvador, BA (2008) 1210-1213.

[8] R. Langer, J.P. Vacanti, Science 2605110 (1993) 920926.

[9] H. Oonishi, Biomaterials 12 (1991) 171-178.

[10] V.S. Gomide, "Desenvolvimento e caracterização mecânica de compósitos hidroxiapatita-zircônia, hidroxiapatita-alumina e hidroxiapatita-titânia para fins biomédicos", Diss. Mestrado, Universidade Estadual de Campinas, S. Paulo (2005).

[11] J. Saggio-Woyansky, C.E. Scott, Am. Ceram. Soc. Bull. 71 (1992) 1674-1682.

[12] V. Chiaverini, Metalurgia do pó - técnicas e produtos,

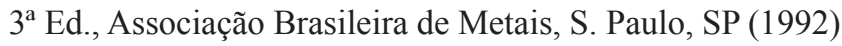
109.

[13] U.U. Gomes, Tecnologia dos pós - fundamentos e aplicações, UFRN Editora Universitária, Natal, RN (1993) 55.

(Rec. 14/12/2015, Rev. 17/04/2016, Ac. 13/06/2016) 